Serviço de Clinica de Doenças Tropicaes e Infectuosas do Prof. Celestino Bourroul

\title{
INSUFFICIENCIA AORTICA TRAUMATICA (I)
}

\author{
Armando K. Cordeiro
}

Quarto annista

Deixando de lado consideraçues de ordem Anatomica e Physiologica do coração vamos passar em revista a pathologia do coraçño.

Na pathologia do coraçăo diśtinguimos as Malformaçōes congenitas e as alteraçōes adquuiridas. Vejamos em primeiro lugar as Malformaçōes congenitas.

Para chegar ao sell desenvolvimento completo n coração experimenta certas transformações, no decorrer das quaes se reprega " tubo cardiaco primitivo e se formam paredes divisorias entre as suas cavidades. A ausencia ou a realizaçăo incompleta de qualquer destes processos faz com que o sér humano nasça com uma malformaçno congenita cardipca.

is malformaçōes congenitas individualizadas na autopsia såo numerosas mas dentre ellas as yue apresentam interesse clinico sĩo em pequeno numero.

Passando rapidamente sobre estas malformaçōes congenitas de interesse clinico nós temos: A molestia de Roger ou Communicação Interventricular, A Persistencia do Buraco de Botal, A estenose da Arteria Pulmonar. A Molestia Azul ou Coração de Fallot, A Estenose ou Coartação do Isthmo Aortico.

Ainda temos nutras malformacies congenitas que deixamos propositalmente de enumerar.

Passando as alteraçöes adquiridas estas sajo. como sabemos das tres camada que formam o coraçăo - o Pericardio, o Endocardio e o Miocardio hem como o seu systema de irrigação, as arterias coronarias. as quaes podem ser séde de alteraçóes pa-

(1) Trabalho apresentado no Departamento sedenufico em 18-5-1937. 
thologicas. Estas sio capazes de comprometter tambem a aort c a arteria pulmonar dada a intima correlaçăo funccional dellas cuin o coraça.

Vejamo, alguma coisa sobre a pathologia destas differentes pertes comesando pelo pericardio.

Como sabemos a inflammaçāo do pericardio denominta-se pericardite e de accórdo com a natureza do exsudato distinguimos: a pericardite fibrinosa, pericardite sero-fibrinosa, a pericardite purulenta e a pericardite hemorrhagica.

Com o nome geral de derrame pericardico englobamos as pericardites filminosa, sero-fibrinosa, purulenta, hemorrhagica, o hidropericardio.

Temos ainda os chamados Pneumopericardios, Hemopericardio, e as associaçőes Hidropneumopericardio, hemopneumopericardio, Piopneumopericardio.

Por hidro-pericardio se entende a transudação de liquido no pericardio, o que se observa nos periodos avançados da insufficiencia cardiaca, e nos estadios pré-mortem (segundo Brur gsch este liquido é produzido talvez durante a agonia).

Passando em seguida a tratar do Endocardio a sua inflam. maçáo constitue a Endocardite e é devido a causas toxico-infegdiosas que evoluem de fórma aguda e sub-aguda e chronica locallzando-se de preferencia nas valvulas produzindo insufficiencis ou estenose, podendo se bcalizar tambem no endocardio parjetal.

A endocardite clinicamente se apresenta sob 2 fórmas: exr docardite simples e endocardite septica; a primeira fórma corres ponde anatomo pathologicamente á trombo endocardite euper. ficial e a segunda fórma à endocardite ulcerosa ou ulcero vegetente.

Brugseh dá para causa ethiologica da endocardite septica ou ulcerosa principalmente o rheumatismo poliarticular agudo, derida principalmente em $59 \%$ dos casos a uma septicemia estreptococcica, em $39 \%$ estaphilococcica, $41 \%$ pneumococcitota, 6 gonococcica e $1 \%$ colibacilar e meningococo de Weichselbaum. agente da meningite cerebro espinhal epidemica. Finalmente póde ainda o endocardio ser séde de processos degenorativos. placas te ateromasia ou transformação fihrosa, fibroser endocardica.

Visto assim rapidamente as Endocardites passamos a vër as Miocardites que sărı as inflammaçōes do miocardio.

Distinguiuse durante muito tempo a miocardite aguda da chronica, hrir em dia ha, em clinica, tendencia sempre crescente a nho se fallar em mirrardite chronica, dada a impossibilidade iratica de a reconhecer nu, leito do doente. 
Quanto á cthiologia das Miocardites, ellas podem ser consequencias de infeçöes agudas sobretudo a diphteria, o rheumatismo poliarticular agudo, a escarlatina, a febre typhoide, tamberm podem ser provocadas pelas infeçóes estreptococcicas. pneumococcicas, meningococcica, grippaes e gonococcicas. Raramente o processo de Miocarlin é isolado, isto $\dot{c}$, sempre um proceso infecciono a que se pode imputar a causa.

O yuadro anatomompathologico da mircardite é representado por precessos exsuclativon e proliferativos do tecido intersticial e nrocessos degenerativos da filira cardiaca.

lim consequencia da lesău de miocardio especializado (ieixe de Hir) apparecem us differentes typos de arithmia: a lesáo do miocarlin commum prosluzirá os differentes gráus de insufficiencia cardiaca.

Denomina-se fibrose micardica nu cardio-cecterone a iran.. formaçåo fibrosa do miocardio. A principio acreditava-se dependesse a fibrose miocardica de intoxicaşo e infecene attenuadas. actualmente ha uma maior tendencia a relacional-a com um "deficit" da irrigaçāo miocardica proveniente de alteraçóes na arvore coronaria. A fibrose do miocardio póde ainda representar a sequella de uma miocardite aguda, sobretudo rheumatica.

Passamos em seguida a Patbologia das arterias coronarias, esta reduz-se praticamente á estenose e á occlusăo total de sua luz com defficiencia na irrigação do musculo cardiaco. Tanto a estenose como a occlusāo podem ser transitorias oul definitivas. Quando sāo transitorias reconhecem como causa um espasmo vascular e náo chegam a determinar alteraçóes estructuraes do miocardio, limitando-se a produzir perturbasöes funccionaes como dör angrinosa, modificaçőes do rythmo, syncope. etc. Quando definitivas a estenose e a occlusão das coronarias resultam de alteraç̆o organica: espessamento da intima por arterio-esclerose, furmaçåo de trombo $\mathrm{cm}$ uma placa de ateroma, embolia, estenoses de orificios de origem por aortite syphilitica, ete.

Si a occlusão c repentina (trombose ou embolia) caso nào se dé a morte immediata. forma-se uma necrose ischemica do miocardin $e$ infarto que logo se transforma numa cicatriz fibrosa é a placa fibrosa post enfartica.

Si a occlusho é lenta por engrossamento gradual da intima c cunsequente reducchio progressiva da luz. produz-ke uma ischemia relativa do miocardio com perturbaçöes funccionacs como syndromo anginosa e transformaçáo fibrosa do miocardio que $\dot{e}$ a fibrose mixcardica primitiva.

A obstruçäo lenta e progressiva do tronco da coronaria D năo traz o exito letal, principalmente em individuos jovens com 
suas: arterias capazes de soffrer uma adaptação e assim vamos vér que a ubstrucção lenta do tronco da coronaria $D_{a}$ sem uma circulaçio colateral, mas uma supplencia, conforme foi verificado experimentalmente com substancias corantes, é feita pela coronaria $E$.

Quando a obstrucçăo é no tronco da coronaria E, a morte wobrevem por falta de irrigação do coração e este é um facto in. teressante a supplencia da coronaria $D$ pela $E$, já não se observando o mesmo quadro quando a obstrucção é no tronco da coro. naria $F$.

Tanto o enfarto miocardico como a placa fibrosa post enfarlica e a fibrose miocardica primitiva por ischemia, representam um ponto de menor resistencia da parede do coração, cuja ruptura ou propulsão para fóra poderá dar-se sob a pressão reinante no interior das cavidades cardiacas e teremos a ruptura expontanea do coração ou o aneurisma parietal do coração.

Quanto ás inflammações da Aorta estas chamam-se Aortopatias, denominando-se mais precisamente de aortites quando inflammatorias e esclerose e ateroma aortico quando degenerativos.

A aortite syphilitica representa a fórma mais bem individualizada, tratando-se de uma pan aortite com destruição da camada média. pois é isto que os allemães denominam de Mesoaortite -1 hilitica, estas circumstancias explicam os differentes typos de incurismas aorticos, estenoses das coronarias na sua origem e inda insufficiencia das valvulas sigmoides, insufficiencia aortica.

limos assim alguma coisa da Pathologia da Aorta, passando \& Arteria pulmonar esta póde soffrer uma inflammaçấo de sua parede constituindo a arterite pulmonar, habitualmente pela syphilis. Tambem aqui se trata uma panarterite com destruiçáo de to cido elastico levanido á dilatação do vaso e mesmo formação do ancurisma. Tambem podemos encontrar a esclerose da arteria pulmonar.

Quando qualquer dessas alterações da art. pulmonar é acompanhada de intensa cianose por hypoventilação alveolár o quaJro clinico corresponde aos casos que Cossio denomina Cardiacos negros de Ayerza. Vejamos em seguida o chamado Augmento do coração: por este nome se denomina o augmento de volume do coraçàn. Podemos distinguir o augmento physiologico, augmento hyperdiastolico, do augmento pathologico ou melhor simplesmente augmento do coração.

$r$ augmento physiologico rep

i maior exigencia physiologico representa o adaptar-se do coracto miocardica alonga-se trabalho, e é uma lei do coraçăo: a fibra ca. Na realidade $n$ auga fornecer uma contracçăo maís energi- 
tole, visto como cada retraç̧áo systolica é mais ampla e faz o orgåo voltar au seu volume primitivo. O augmento cardiaco isto $\dot{e}$ o augmento pathologico de volume $\dot{c}$ permanente: o curaçáo tem maior tamanho tanto na diastole como na tyatole. Nesse caso trata-se de adaptaçäo do urgüu, nâo mais a uma mair.r exigencia circulatoria, mas a uma retraçán systolica cuja amplitude é menor em virtude da insufficiencia do poder contratil the mineardio. Cum effeito como acontece a todo corpu espherico a capacidade das cavidades esta em relação com " cubu de =el raiu. İestas cintdiçoes "a augmentu de tamanhu das cavidades cardiacas permitte que com maior encurtamento do raio se expulse a mesma quantidade de sangue que anteriormente.

() augmento cardiaco é devide a dilatacáo das cavidades, diLitaçà cardiaca. C augmento da espessura do miocardiu. hypertrophia do miccardio. Ein geral ambos participam do processo pendendo algumas vezes haver preiluminancia de um ou outro.

Na clinica nä, ha signaes que permittan distinguir hypertrophia da dilatação, mo augmento de wulume cardiaco é preferivel fallar apenas (II) augmentu cardiaco e nis hypertrophia oll dilataçä). Quandu o augmento se produz repentinamente como acontece 11 m. csíurço excessivu, dilataçào aguda do coração ou num atapue prolungarlo de tachicardia paroxistica so esta cm jogo um processo de dilataçăo.

O augmento do curaçio pirle limitar-se a um ou dois ou a todos compartimentos do curação. Em geral no inicio "a augrnen10 Carliaco fica restricto ao segmento que é séde do transtomo mechanicu á circulaçäo do sangulc, mas logo se extende aos temais segmenfus, dada a repercussäo que nelles tem " transtorno inicial.

Av miucardites anudas e super-agudas occasionam um augmento total do curaçäo.

Passamos em seguida a estudar as Arithmias.

Arithmias sino as perturbagies do rythmo: augmente ou diminuçăo da frequencia, irregularidades. tran-tornos da conduecăn a alternancia. Segundu Vayjue e Donzeint é actualmente impossivel uma clasciificasao pathengenica la-arithmias, de mulo que so e possivel uma classificaçü puramente clinica poderá ser aduptada.

Para facilidade didactica podemos dividir em 2 categurias or processus reymensaveis pelas alteracies do rythmo cardiaco: 1) ... que irritam e w que inhihem as proprielades do mincardio. e de accórdo com a regiono miocurlica em que prothe a irritaçin … a inhibican. ter.er á ceta ou aquella desordem do ryttmo.

Vejami.. as Arrithmias por imitaçäo: 
Os processos irritativos exaltam as propriedades do miocardio especifico. Si a irritação se processa de uma maneira continua apparece a tachicardia, si ella se faz de quando em quando temos as extrasistoles.

Tachicardia é a acceleração dos batimentos cardiacos, distinguindo-se a tachicardia sinusal e a heteropica ou paroxistica, a differença é que uma parte do miocardio auricular gera o estimulo.

Passamos em seguida ás Extrasistoles são contracções prematuras que interrompem a cadencia dos batimentos cardiacos ou estimulos que dão lugar ás contracções, 'que cahem fóra do rythmo normal do coração. O lugar onde nasce a extrasistole corresponde ao ponto de irritação do miocardio, assim temos: as extrasistoles sinusaes, auriculares, nodaes ou auriculo-ventriculares e ventriculares.

A inhibição deprime as propriedades do systema excito conductor, si ella actua sobre o nodulo sinusal predomina a diminuição do automatismo e' se agem sobre o resto do miocardio especializado preponderam os transtornos de conducção auriculo ventricular.

A depressão permanente das propriedades do nodulo sinusal occasiona ou a bradicardia sinusal ou o rythmo nodal conforme o gráu de inhibição.

A depressão intermitente do automatismo do nodulo sinusal acarreta o aborto sinusal, chamado bloqueio sino auricular. Si a depressão se localiza no feixe de His, temos alteração da conductibilidade auriculo ventricular e conforme o gráu de depressã̃o temos ou o augmento do tempo de conducção ou o bloqueio. Quando a depressão - é ligeira temos augmento de tempo de conducção auriculo ventricular. Si a depressão é mais accentuada temos interrupção parcial ou total da conducção do estimulo das auriculas para os ventriculos e temos bloqueios parciaes ou incompletos e os bloqueios completos ou totaes respectivamente.

Si a depressão se localiza em um dos ramos do feixe de His ha alteração da conducção intra-ventricular constituindo o thamado bloqueio de ramo e como consequencia a excitação dos 2 ventriculos não é simultanea, contrae-se primeiro o que tem seu ramo intacto e depois o lesado, por propagação do septo intra-ventricular.

Passando em seguida as arithmias que produzem ao mesmo tempo irritação e inhibição no systema excito conductor temos a arithmias respiratoria, a arithmia sinusal, a interferencia dos 2 rythmos, o flutter e fibrilação auricular.

Segundo Vieira Romeiro distinguimos nas arithmias:

1) As arithmias sinusaes.

2) As Extrasistoles. 
3) A Arithmia completa, Fibrilação auricular.

4) O pulso alternante.

5) As tachicardias.

6) As bradicardias.

Nas arithmias sinusaes considera ainda este A.:
a) Tachicardias sinusaes.
b) Bradicardias sinusaes.
c) A. arithmia respiratoria.
d) Pulso paradoxal.

Nas extrasistoles considera ainda: extrasistoles sinusaes, auriculares, ventriculares e nodaes. Podendo ainda ser irregulares e isoladas, rithmadas e agrupadas.

Nas tachicardias considera as Tachicardias paroxisticas e não paroxisticas. As tachicardias não paroxisticas são: phisiologicas (trabalho muscular, ortostatica) e pathologicas como nos estados febris, anemicos e caqueticos, intoxicações, affecções cardiovasculares e 'outros estados pathologicos.

As tachicardias paroxisticas distingue 2 typos: a tachicardia paroxistica total 'ou doença de Bouvet e a fórma parcial ou Flutter dns Inglezes.

Von Bergmann descreve as anomalias nomotopas de formação do estimulo que são: Tachicardia sinusal, Bradicardia sinusal, Arithmia sinusal, Arithmia respiratoria e a juvenil. As anomalias heterotopas: Arithmia extrasistolica, arithmia absoluta e os transtornos na conducção, são os differentes typos de bloqueio, ainda descreve os transtornos da contractilidade do coração.

Uma vez vista assim de conjuncto, muito rapidảmente a pathologia do coração, vejamos o que se entende por Insufficiencia circulatoria.

Como sabemos o apparelho circulatorio tem por funç̧ão movimentar a massa sanguinea que serve de vehiculo ao oxygenio e ás substancias nutritivas necessarias á manutenção da vida dos differentes tecidos. Quando esta mobilização se processa de uma maneira defficiente, diz-se que ha insufficiencia circulatoria. Em geral está cheio o systema venoso e relativamente vazio o systema arterial. Ésta distribuição anomala do sangue é o phenomeno de conjuncto mais nòtavel na Insufficiencią circulatoria. Além disso, sua generalização é tambem parte essencial do quadro clinico, já que a extase local, como se observa, por, exemplo, na trombose venosa, não é insufficiencia circulatoria.

Como sabemos ha 2 classes de insufficiencia circulatoria: uma central ou cardiaca e a outra de origem peripherica ou vascular, a 
primeira é conhecida com o nome de insufficiencia cardiaca e a segunda com o nome de insufficiencia peripherica. Estas duas classes $\mathrm{de}$ insufficiencia podem existir isoladamente ou associadas. Em geral, a principio se inicia a insufficiencia circulatoria por um desses typos, porem mais tarde os dois se associam e não sendo raro ver uma insufficiencia cardiaca complicada por uma insufficiencia peripherica e o contrario tambem se observa.

Vejamos em primeiro lugar a' Insufficiencia cardiaca.

Diz-se que ha insufficiencia cardiaca quando o coração perde a capacidade de fornecer ao organismo a quantidade de sangue necessario, a massa liquida circulando mais lentamente se accumula em diversos territorios e o plasma póde transudar e infiltrar os intersticios cellulares.

A defficiencia do rendimento do orgão circulatorio resulta ou da insufficiente evacuação systolica ou da incompleta repleção dias-: tolica. Em certas condições' especiaes os 2 mechanismos se associam para dar origem ao "deficit" circulatorio como se observa na estenose mitral e adherencias pericardicas.

A evacuação systolica defficiente reconhece como causa a diminuição do poder contractil do miocardio seja por lesão (miocardite, etc), seja por um excesso de trabalho (porque provoca a diminuição do poder contractil do miocardio, em virtude da distensão exaggerada das fibras cardiacas ou do proprio processo de hypertrophia que elle determina).

A incompleta repleção diastolica das cavidades cardiacas tem por causa a compressão do orgão (derrame pericardico) ou uma grande acceleração cardiaca com consequente diminuição do repouso diastolico (tachicardia paroxistica).

A evacuação systolica ou a repleção diastolica defficiente isoladas ou combinadas determinam uma série de disturbios hemodinamicos. O volume systolico diminue e embora haja acceleração dos batimentos cardiacos o volume circulatorio na unidade de tempo soffrerá gráus variaveis de diminuição. A vis a tergo reduz-se e o sangue circulando mais lentamente estagna-se ao nivel dos-tecidos aos quaes abandona uma maior quantidade de oxygenio. Em circumstancias especiaes a insufficiencia cardiaca reduz a vis a tergo e augmenta a "vis a fronte" em virtude do accumulo da massa liquida no systema venoso. A pressão hydrostatica augmentará então ao nivel dos capillares havendo transudação do plasma, que infiltrará os intersticios cellulares, é a insufficiencia

O gráu mais adiantado da insufficiencia cardiaca traduz-se pela existencia de um "deficit" circulatorio mesmo quando são 
minimas as exigencias organicas, como por exemplo no repouso absoluto.

A escola franceza tem procurado differenciar dois typos de insufficiencia cardiaca chronica: a insufficiencia do V. E. e a insufficiencia do V. D.

Vejamos a seguir a Insufficiencia neripherica, neste caso a "deficit" circulatorio corre por conta de um retorno incompleto do sangue ao coração. Como sabemos o systema vascular tem capacidade superior ao volume da massa liquida circulante no seu intẹrior graças á acȩão dos nervos vaso motores e ao reflexo de Hess: no orgão em actividade ha vaso dilatação ao passo que nos orgãos em repouso domina vaso constricção e como consequencia o continente se adapta ao conteudo, de modo que não haja estagnação do sangue, que retorna totalmente ao orgão central da circulação.

Si como acontece nos estados toxi-infecciosos graves, traumatismos violentos, hemorrhagias, etc., a vaso dilatação de extensos territorios vasculares, impede essa adaptação do continente ao conteudo, o sangue estagna, e não retorna na sua totalidade ao coração. A repleção diastolica passa a ser insufficiente e - coração lançará na arvore arterial uma quantidade de sangue menor, estabelece assim um circulo vicioso ao qual sobrevem a insufficiencia circulatoria."

A essa insufficiencia circulatoria podem juntar transtornos vaso motores cutaneos com sudação profusa com resfriamento geral e lividez das extremidades constituindo o colapso algido que vem agravar ainda mais a situação em virtude de subtrahirem liquido.

Quando sobrevem insufficiencia circulatoria peripherica repentinamente após intervenções cirurgicas, traumatismo temos o shock operatorio ou traumatico. Vimos assim rapidamente a insufficiencia circulatoria com seus 2 typos: insufficiencia cardiaca e insufficiencia peripherica. Interessa-nos mais de perto a insufficiencia cardiaca e desta a insufficiencia aortica.

A insufficiencia aortica caracteriza-se clinicamente por uma série de signaes physicos de que veremos os mais importantes. A insufficiencia aortica é uma das cardiopathias mais frequentes nesta afecção: o sangue reflue na diastole, da aorta para o V. E. que ao mesmo tempo recebe o sangue que vem da A. E. Assim recebendo maior quantidade de sangue este segmento do coração se dilata e depois se hypertrophia que quando é definitiva compensa a lesão.

Anatomo pathologicamente temos na insufficiencia aortica:

1) Dilatação do cone aortico. 
2) Dilatação do segmento apical com redondamento da ponta.

3) Achatamento dos musculos papillares e trabeculas.

4) Hypertrophia secundaria da musculatura ventricular, principalmente dos musculos papillares devido ao augmento da tensão valvular pela dilatação do annel da valvula mitral.

5) Dilatação secundaria e hypertrophia da aorta.

Vejamos então os signaes physicos:

1) Signaes centraes ou cardiacos: nestes temos:
a) Choque da ponta cupuliforme ou "endome" de Bard, ou globoso.
b) Fremito pre systolico de Flint.
c) Inspecção e palpação revela deslocamento do ictus para baixo e para fóra, para a esquerda.
d) Percussão confirma a inspecção e palpação isto é, o augmento de volume do coração.
e) Sopro diastolico mais audivel na parte média do externo.
f) Ruflar de Flint que é audivel na ponta é um sopro pre-systolico, sem nenhuma lesão organica.

2) Signaes periphericos ou arteriaes periphericos:
a) Pusatilidade anormal das carotidas, sub-clavias axilares, humeraes, etc. (dansa das arterias).
b) Pulsatilidade da aorta na furcula.
c) Palpação na aorta na furcula.
d) Fremito da aorta e outros vasos.
e) Pulso Corrigan ou em martelo de agua.
f) Signal de Musset (inclinação da cabeça rythmica com as contracções systolicas.
g) Pulso popliteu.
h) Pulso capillar de Quincke, verificado nas unhas e nos labios.
i) Pulso da uvula ou signal de Müller, são movimen- tos oscillatorios da uvula.
j) Pulso amygdalo-carotidiano, signal de Huchard.
k) Pulso da lingua ou signal Minervini, consiste na elevação da lingua em cada systole.
1) Signal de Landolfi, estreitamento da pupilla na systole e da dilatação na diastole.
m) A auscultação da carotida revela o desapparecimen- to do $2 .^{\circ}$ tom.


n) Signal de Alvarenga-Duroziez.

o) Duplo tom de Traube.

p) Pistol-shot, nas pediosas.

Ainda além destes signaes devemos fazer esphigmograma, electrocardiogramma, radiographia do coração e vasos da base, bem como medirmos a tensão arterial cuja differença é caracteristica.

Deve-se ainda fazer reacção de Wassermann, pesquizar o reflexo patelar e o signal de Argyll-Robertson para a possibilidade de un diagnostico de Tabes incipiente, que Babinsky diz não ser raro junto á insufficiencia aortica.

Quanto aos typos de insufficiencia aortica os mais importantes são em numero de quatro: 1) a insufficiencia aortica por ruptura valvular (traumatismo). 2) A endocardica (Doença de Corrigan). 3) A de origem arterial (Doença de Hogdson) e 4) Insufficiencia aortica funccional, isto de accôrdo com Vieira Romeiro.

$\mathrm{Na}$ insufficiencia aortica por ruptura valvular, as desordens funccionaes são subitas, logo após a lesão valvular. Observa-se depois de um violento esforço, de um traumatismo violento sobre - thorax, de uma queda de grande altura, os doentes sentem uma viva dôr na região pre-cordial e em seguida violenta dispnéa que muitas vezes o individuo perde os sentidos.

A insufficiencia aortica endocardica ou doença de Corrigan apresenta como causa mais frequente o Rheumatismo poli-articular agudo, e a insufficiencia aortica de origem arterial ou doença de Hogdson, é geralmente devida á Syphilis.

$\mathrm{Na}$ insufficiencia aortica funccional não ha lesão do apparetho oro-valvular.

Destes 4 typos de insufficiencia aortica interessa-nos o primeiro isto é, a insufficiencia aortica por ruptura valvular.

A insufficiencia aortica por ruptura valvular como dissemos é verificada após traumatismos, violentos sobre o thorax como seja quéda de grande altura, esforço violento. Quanto a ethiologia clinica desta molestia estamos de accôrdo com Scherf, e que diz que uma fórma relativamente rara é a insufficiencia aortica traumatica. E' encontrada quasi sempre com uma mesoaortite. Póde apparecer acompanhando os effeitos de uma elevação brusca da pressão sanguinea, pela existencia de um ponto ordinariamente já adoecido,

Ouve-se um tom musical de um sopro, que acompanha ao mesmo tempo a bulha diastolica. Muito frequentemente nos adultos, por muito tempo sentem uma dôr persistente. 
Uma vez vista assim alguma coisa sobre a pathologia do coração vejamos o caso verificado por nós na 6. ${ }^{a}$ Medicina de Homens da Santa Casa, Serviço do Prof. Celestino Bourroul, deixando aqui nossos agradecimentos aos Drs. Cicero de Moraes e Oscar Barcellos, o primeiro por nos ter suggerido a apresentação deste caso e que muito nos orientou, bem como o Dr. Oscar Barcellos.

T. S. - 46 ANNOS - VIUVO - LAVRADOR - JAPONEZ - AMARELLO - CAPITAL.

QUEIXA E DURAÇÃO: Dôr na região pre-cordial após um coice de burro, dispnéa, ha 4 mezes.

H. P. M. A.: Ha 4 mezes, lidando nos trabalhos ruraes recebeu um coice de burro no hemithorax $E$, na parte anterior correspondente á região cardiaca e outro no hypocondrió D. 0 traumatismo foi tão violento que teve de se acamar durante 15 dias, apparecendo-lhe logo em seguida falta de ar, não podendo por isso fazer qualquer esforço violento ou andar grandes distancias. Ao mesmo tempo sente dôr na região pre-cordial no ponto em que soffreu o traumatismo.

Sempre foi forte e trabalhava com disposição antes de soffrer o traumatismo.

Ha cerca de um mez teve grippe acompanhada de febre e tosse com escarro de côr branca sem şangue. Desde então vem o seu estado se aggravando dormindo mal devido á dispnéa que o obriga a procurar a janella ou a posição orthopneica, sentindo constantemente dôr no ponto em que soffreu o traumatismo correspondente a região pre-cordial.

Nega qualquer alteração para o lado dó apparelho urinario. tancia.

Interrogatorio sobre os differentes apparelhos: Sem impor-

\section{Antecedentes pessoaes}

Doente nascido em condições normaes passando uma infancia sadia. Trabalha em serviço pesado (enxada), alimentando-se bem, não fazia exercicio matinal, fumava regularmente (15-20 cigarros por dia), bebia bastante, meio litro de canninha diariamente, desde os 18 annos até a edade de 44 annos. Aos 20 annos teve uma perturbação gastro-intestinal. Nega molestias proprias da infancia, rheumatismo, angina e outras molestias infecciosas. Nega antecedentes venereo-syphiliticos.

\section{Antecedentes familiares e hereditarios}

Paes fallecidos: Pae fallecido de molestia do figado (sic). Ignora a causa da morte da mãe. Tem um irmão vivo e forte. 
E' viuvo a esposa fallecida de Dysenteria amebiana. Tem 2 filhos vivos e fortes. Negando abortos ou nati-mortos.

\section{Exame objectivo}

Individuo de compleição robusta, typo brevilineo (Charpy maior que $90^{\circ}$ ) apresentanido physionomia calma, quasi indifferente ao meio. Não apresenta dispnéa nem edema. Pelle humida e quente de côr caracteristica da raça a que pertence, não apresentando cicatrizes, nem circulação collateral. Mucosas visiveis ligeiramente descoradas. Paniculo adiposo regular e bem distribuido. Musculatura bem desenvolvida. Arcabouço osseo bem conformado. Mucosas externas ligeiramente descoradas. Pellos com disposição masculina.

Mede 1,65 de altura e pesa 58 kilos.

Systema ganglionar: ganglios cervicaes e inguinaes moles, moveis sobre os planos subjacentes e indolores.

\section{Exame especial}

O mais interessante é o exame do pescoço.

Pescoço: fino e cylindrico, apresentando saliencia dos musculos esterno-cleido-mastoideus, notando-se batimentos arteriaes sinchronos com os batimentos cardiacos. Apresentando-se estas arterias do pescoço duras, ateromatosas.

\section{Thorax}

Inspecção estatica F. A.: Thorax chato, simetrico, apresentando hombros na mesma altura, claviculas ligeiramente salientes, fossas supra e infra-claviculares, pouco escavadas. Mammilos na mesma altura e a igual distancia da linha médio-esternal. Intercostos visiveis principalmente os ultimos. Charpy maior que $90^{\circ}$. Louys visivel.

F. P.: Hombros na mesma altura, fossas supra e infra-espinhosas muito excavadas, espaço inter-escapulo vertebral ligeiramente excavado, bordos espinhaes dos homoplatas, ligeiramente salientes, e situados a igual distancia da linha espondilea. Angulos inferiores dos homoplatas situados na mesmá altura e a igual distancia da linha espondiléa.

Inspecção dinamica: Typo respiratorio thoraco abdominal, movimentos amplos e iguaes de ambos os apices e bases, Litten visivel de ambos os lados, seguindo uma linha parallela a $2 \mathrm{cms}$. do rebordo. Schnup. ph.: visivel de ambos os lados. Sprechen. ph.: idem. Lemos Torres: negativo. 
Palpação: Fremito thoraco vocal: Normal.

Percussão: Som claro pulmonar em toda área.

Ausculta: Murmurio vesicular normal em toda a area pulmonar.

Apparelho Cardio-Vascular: Coração: Inspecção: Ictus batendo no $6 .^{\circ}$ espaço intercostal $\mathrm{E}$, uns $3 \mathrm{cms}$., para fóra da linha mammilar, para baixo e para a E. Palpação: Confirma a inspeç̧ão e demonstra fremito precordial. Percussão: Área cardiaca augmentada de volume. Ausculta: Revela sopro diastolico no fóco aortico propagando-se para cima ao longo do esterno. Duplo sopro, no fóco mitral. Arterias moles, elasticas e depressiveis, pulso batendo 108 vezes por minuto, regular em tempo e amplitude. Pressão maxima 135 e pressão minima 80 . As arterias do pescoço são duras, ateromatosas.

\section{Abdomen}

Nada digno de nota.

\section{Columna e reflexos}

Nada digno de nota.

Pesquizas dos signaes de Insufficiencia Aortica. Signaes centraes:

1) Choque da ponta Cupuliforme: positivo.

2) Fremito pre-systolico de Flint: positivo. cardiaca.

3) Percussão do coração e áorta: revela aúgmento da área

4) Sopro doce, diastolico no fóco aortico com propagação para cima ao longo do esterno.

5) Ruflar de Flint:/__

Signaes periphericos:

1) Pulsatilidade das carotidas, sub-clavias, axillares, humeraes (dansa das arterias): positivo.

2) Pulsatilidade da aorta na furcula: positivo.

3) Palpação da aorta na furcula: positivo.

4) Fremito da aorta e outros vasos. /

5) Pulso Corrigan: positivo.

6) Signal de Musset: positivo.

7) Pulso popliteu: não foi pesquizado.

8) Pulso capillar de Quincke (labios e unhas) /

9) Pulso da uvula (Müller) /

10) Pulso amigdalo carotidiano. / $/$

11) Pulso da lingua ou signal de Minervini 
12) Signal de Sandelfi (iris: estreitamento na systole e dilatação na diastole.

13) Auscultação da carotida (desapparecimento do $2 .^{\circ}$ tom) não foi feito.

14) Signal de Alvarenga Duroziez: positivo.

15) Duplo tom de Traube. /

16) Pistol shot nas pediosas. /

17) Tensão arterial: $135-80$.

\section{Exames complementares:}

Reacção de Wassermann no sangue: negativa.

Exame de urina: Vol 275 cc. Dens 1.034. Albumina: contem - Assucar: contem. Exame microscopico: normal.

ELECTROCARDIOGRAMA: Desvio do eixo para a direita. $Q R \mathrm{R} 1$ espessada e $\mathrm{T} 1$ pequeno.

RADIOGRAPHIA : do coração e vasos da base: Area cardiaca medindo approximadamente $200 \mathrm{~cm} .2$, apresentando, portanto, um excesso de mais de $100 \%$ sobre a área normal para o peso. Configuração radiologica de augmento predominante do ventriculo esquerdo. Aorta medindo approximadamente $40 \mathrm{~mm}$. de diametro ao nivel da crossa. Accentuada extase pulnłonar. Fócos esparsos de condensação no pulmão D.

\section{Evolução e tratamento:}

Em $10^{\circ}$ de abril durante o decurso de sua permanencia nesta enfermaria appareceram edemas generalizados, mais accentuados na face, dispnéa, tosse com escarros mucosos. Medida a pressão arterial esta nos deu Mx. 145 e Mn. 80. Pulso batendo 134 vezes por minuto. A percussão do thorax encontramos sub-massicez nas bases e a ausculta revelou: estertores sub-crepitantes finos e alguns sibillos. Feito exame de urina revelou grande quantidade de albumina.

O tratamento consistiu:- em sangria $300 \mathrm{cc}$., ventosas sarjadas nas bases, 30 gottas de Digitalina e Salirgan. Passado o perigo do edema agudo do pulmão, o estado do paciente se normaliza e a lesão cardiacł entra em periodo de compensação.

Durante sua estada nesta enfermaria o doente tomou 90 gottas de Digitalina, "3 ampo!las de Salirgan, Salicilato de sodio e Iodeto de Potassio. A 17 de abril o doente pediu alta em condições relativamente bôas. 
Portanto como vemos, este caso, é um caso de Insufficiencia aortica em que a ethiologia poderá ser explicada pelo alcoolismo ou pela ateromazia das valvulas sigmoides aorticas (?), e a causa desencadeadora foi o traumatismo (no caso apresentado coice de um burro).

Brugsch - Prof. Dr. Theodor - Tratádo de Pathologia Medica. Traducção hespanhola. Edit. Labor S. A., 1934.

Cossio-Pedro - Coração e Vasos (Semiologia). Editora Nacional - 1937.

Romeiro-Vieira - Semiologia Medica - 1933.

Scherf-D. Klinik und Therapie der Herzkrankheiten und der Gefässer-korankugen - 1936.

Von-Bergmann - Tratado de Pathologia Medica - 1936. 